\title{
PURIFICATION, CHARACTERIZATION AND SEQUENCING OF AMYLASE A FROM ALKALIPHLIC BACILLUS LICHENIFORMIS STRAIN-MK7
}

\author{
Mohamed kasem ${ }^{1}$, Aysam M. Fayed ${ }^{2}$, Samah Eldemery², Adel Okba ${ }^{1}$ and Atef Ibrahim ${ }^{1}$
}

1- Microbial biotechnology dept. 2- Molecular Biology dept., Genetic Eng. and Biotech. Research Inst., University of Sadat City (USC), Egypt

Corresponding author: atef.ibrahim@gebri.usc.edu.eg

\begin{abstract}
Two degenerative primers were designated from the closest Bacillus, Bacillus licheniformis strain DSM13 (ATCC 14580) which has complete genome to get amylase gene from our Bacillus licheniformis strain MK7 (KP322016) by PCR technique. We got about $1500 \mathrm{bp} \mathrm{PCR}$, the sequence analysis revealed that the alkaliphilic cellulase belongs to glycosyl hydrolase family $12 \mathrm{~A}$ which has $100 \%$ identity to glycoside hydrolase from Bacillus sp. (WP009329478) and has $99 \%$ identity to family 12 xyloglucanase from Bacillus licheniformis (2JEMIA). The nucleotide sequence of the alkaliphilic cellulase gene (cel12A) was deposited to the GenBank under accession number (KP322015). The cel12A gene from B. licheniformis strain MK7 has open reading frame of $1500 \mathrm{bp}$ that codes for 449 amino acid which has a molecular weight of 55KDa protein. The enzyme protein was further purified using ammonium sulphate precipitation and chromatography. The characterization of amylase protein showed that the enzyme has an optimum temperature at $\left(37^{\circ} \mathrm{C}\right)$, optimum $\mathrm{pH}(9.0)$ and Some metals increase the enzyme activity such as $\mathrm{CoCl}_{2}{ }^{+}, \mathrm{K}^{+}$and EDTA, but others decrease the activity such as $\mathrm{Ag}^{+}, \mathrm{Zn}^{2+}$ and urea ions.

Keywords: Amylase A, Purification, characterization, Bacillus licheniformis strain -MK7.
\end{abstract}

\section{INTRODUCTION}

Alkaliphillic bacteria are defined as organisms that grow optimally at alkaline $\mathrm{pH}$, with $\mathrm{pH}$ optima for growth being in excess of pH 8 (usually between 8 and 10), and some being capable of growing at $\mathrm{pH}$ higher than 11 (Grant and Jones, 2000; Horikoshi, 1999). Alkaliphillic bacteria are reported to be a rich source of alkaline active enzymes e.g. amylase, cellulase, xylanase, and other enzymes which have numerous applications in industrial processes (Horikoshi, 1991). Among the bacteria, the genus Bacillus is an attractive industrial organism due to growth rate, extracellular secretion and biosafety capacity (Schallmey et al., 2004). Amylases, glycoside hydrolases, break starch into glucose, maltose, maltotriose and dextrin by hydrolysis of glyosidic bonds. Therefore they are also called digestive enzymes. The first enzyme produced industrially was amylase from a fungal source in 1894, which was used as a pharmaceutical aid for the treatment of digestive disorders (Pandey et al., 2000). Amylases are obtained from various origins like plant, animal, bacterial and fungal. Several researchers produced amylases enzyme using Bacillus sp. (Yuguo et al., 2000; Young et al., 2001; Dharani, 2004 and Zambare, 2010a). Amylase has many applications in food, textile, paper and pulp,

pharmaceuticals, baking and beverages, detergent and leather industries (Pandey et al., 2000; Reddy et al., 2003a and Kar et al., 2010). Amylases constitute a class of industrial enzymes, which alone form approximately $25 \%$ of the enzyme market covering many industrial processes such as sugar, textile, paper, brewing, distilling industries and pharmaceuticals (Mamo et al., 1999; Oudjerouat et al., 2003; Pandey et al., 2000). Amylases are classified into two categories; Endoamylases and Exoamylases (Gupta et al., 2002). Endoamylases catalyze hydrolysis in a random manner in the interior of the starch molecule. This action causes the formation of linear and branched oligosaccharides of various chain lengths. Exoamylases hydrolyze from the non-reducing end, successfully resulting in short end products. The production of microbial amylases from bacteria is dependent on the type of strain, composition of medium, method of cultivation, cell growth, nutrient requirements, incubation period, $\mathrm{pH}$, temperature, metal ions and thermos ability (Pandey et al., 2000). In fact, such industrially important microorganisms found within the genus Bacillus, can be 
exploited commercially due to their rapid growth rate leading to short fermentation cycles, capacity to secrete proteins into the extracellular medium and safe handling (Pandey et al., 2000). The most widely used thermostable amylases in the starch industry are produced from $\boldsymbol{B}$. licheniformis (Morgan et al., 1981). Microbial production of amylase is more beneficial than other sources because it is economical; production rate is high and can be engineered to obtain enzymes of desired characteristics. The microbial amylases could be potentially useful in various pharmaceutical, fine-chemical industries, paper industries etc. With the emergence of biotechnology, the use of amylase has widened in clinical research, medical chemistry and starch analytical chemistry. These increased uses have placed greater stress on increasing indigenous amylase production and search for more efficient processes (Aiyer et al., 2005).

Present study is related to the isolation, characterization and sequencing of amylase producing bacteria from the soil samples collected from the fields of Sadat city, (Menoufiya governerate, Egypt.

\section{MATERIALS AND METHODS}

\section{Isolation of bacterial cultures}

Soil samples were collected from field of Sadat city (menofya), Egypt from 3 to $4 \mathrm{~cm}$ depth then transferred into sterile plastic bags and maintained in aseptic conditions. Isolation of soil bacteria was performed by serial dilution and spread plate method as described earlier (Aneja, 2003). A volume of $30 \mu \mathrm{l} \mathrm{ml}$ of each dilution was transferred aseptically to starch agar plates. The sample was spread uniformly. The plates containing Starch $0.5 \%$, Trypton $1.5 \%$, Nacl $20.5 \%$, Agar $1.5 \%$, $\mathrm{Na} 2 \mathrm{CO} 31 \%(\mathrm{w} / \mathrm{v})$, the $\mathrm{pH}$ was adjusted to 9.5 with $\mathrm{Na}_{2} \mathrm{CO} 3(10 \%, \mathrm{w} / \mathrm{v})$ then incubated at $37^{\circ} \mathrm{C}$ for $24 \mathrm{hr}$. The bacterial isolates were further sub cultured to obtain pure culture. Pure isolates on starch agar slants were maintained at 4 으.

\section{Genomic DNA isolation}

Genomic DNA of the two amylolytic Bacillus licheniformis strain-mk7 was extracted from cells grown on LB broth according to the protocol for bacterial DNA extraction with the Gene JET Genomic DNA Purification Kit (\#K0721) provided by Thermos Scientific.
Genomic DNA fragment encoding amylase (AmyA) was amplified by PCR with chromosomal DNA of Bacillus licheniformis strain-MK7 as a template and two degenerative oligonucleotide primers: Ksm- $\mathrm{F}$ GGATCCATGAAACAACAAAAACGGCTTTAC and Ksm-R1GGATCCCTATCTTTGAACATAAATTGAAAC and Ksm-R2 GGATCCTTCTGCTGCGACATCAGGATGGTC were synthesized based on the nucleotide sequence of closest Bacillus licheniformis strain DSM13 (ATCC 14580) amylase.

\section{DNA Sequencing of amylase gene}

Nucleotide sequencing was carried out on applied Bio systems model 373 A ( Lincoln, Nebr ) model 4000L automated DNA sequencer with appropriate dye primers ( Macrogen Inc. Souel, Korea). The nucleotide sequence data was analyzed with GENETYX computer software (Software Development Co. Itd., Tokyo, Japan). Homology searches in Gene Bank were carried out with a Blast Program (Kenji et al., 1996).

\section{Enzyme assay}

The isolate which showed high zone of clearance were used for further studies. Freshly prepared inoculum was used to inoculate the production medium. $500 \mathrm{ml}$ of the production medium was inoculated with $10 \mu$ of bacterial inoculum. The flask was loaded on a rotary shaker incubator at a speed of $2000 \mathrm{rpm}$ at $37^{\circ} \mathrm{C}$ for 24 hours. After incubation, broth was centrifuged at $10000 \mathrm{rpm}$ for $15 \mathrm{~min}$ was collected and used for the estimation of amylase. Purification of amylase enzyme was achieved by ammonium sulphate precipitation followed by dialysis. $100 \mathrm{ml}$ of cell free extract was saturated with ammonium sulphate up to $80 \%$. The content was incubated overnight and centrifuged at $6000 \mathrm{rpm}$ for $20 \mathrm{~min}$ Amylase was determined by spectrophotometric method as described by Fisher and Stein (1961). According to the procedure $1.0 \mathrm{ml}$ of culture extract 'enzyme' into a test tube and $1 \mathrm{ml}$ of $1 \%$ soluble starch in phosphate buffer ( $\mathrm{pH} \mathrm{9.0)} \mathrm{was}$ added in test tube. The test tubes were covered and incubate at $35^{\circ} \mathrm{C}$ for 15 minutes in water bath. Then 2.0 $\mathrm{ml}$ DNS reagent was added in each tube to stop the reaction and kept in boiling water bath for 5 minutes. After cooling at room temperature, the absorbance was read at $540 \mathrm{~nm}$ by spectrophotometer (Fisher and Stein, 1961).

\section{PCR amplification of alkaliphilic amylase gene}




\section{Enzyme characterization}

\section{Optimum temperature}

The activity was determined by carrying out the assay at several temperatures. $100 \mu$ of appropriate concentration of enzyme were added to $100 \mu \mathrm{l}$ of $1 \%$ starch and completed to $1 \mathrm{ml}$ with $800 \mu \mathrm{l}$ of $20 \mathrm{mM}$ glycine- $\mathrm{NaOH}$ buffer ( $\mathrm{pH}$ 9.0), and incubated at various temperatures $(10,15,25,37,50$, and 70$)$ for $30 \mathrm{~min}$. The activity was then measured according to (Miller, 1959).

\section{Effect of $\mathrm{pH}$ on amylase $\mathrm{A}$ activity.}

Buffer $(0.05 \mathrm{M})$ of different $\mathrm{pH}$ ranging from 5.0, 5.5, 6.0, 6.5, 7.0, 7.5, 8.0, 9.0, 9.5, 10.0 were prepared using different buffer system. Each of this buffer solution was used to prepare $1 \%$ soluble starch solution used as substrate in assaying the enzyme as the following $100 \mu \mathrm{l}$ of appropriate concentration of enzyme were added to $100 \mu \mathrm{l}$ of $1 \%$ starch and completed to $1 \mathrm{ml}$ with $800 \mu \mathrm{l}$ of various buffers then incubation at $37^{\circ} \mathrm{C}$ for $30 \mathrm{~min}$, add 1 $\mathrm{ml}$ of DNS into the mixture. The assay was carried out according to standard assay procedure measured the activity according to (Miller, 1959).

Effect of Heavy Metals on Enzyme Activity.

For activators and/or inhibitors sensitivity studies, the enzyme was pre-incubated with a final concentration of $1 \mathrm{mM}$ of various reagents $(\mathrm{Ag}+, \mathrm{K}+, \mathrm{Co} 2+, \mathrm{Fe} 3+, \mathrm{Mg} 2+$, $\mathrm{Cu} 2+, \mathrm{Zn2+}$, EDTA, Urea) dissolved in $50 \mathrm{mM}$ glycine$\mathrm{NaOH}$ buffer ( $\mathrm{pH} 9.0)$ at $370 \mathrm{C}$ for $30 \mathrm{~min}$. (Oda et al., 1993) .The residual activity was measured by using the standard assay conditions.

\section{RESULTS AND DISCUSSION}

PCR product of alkaliphilic amylase gene from $B$. licheniformis strain-MK7

PCR amplification was performed as mentioned before (materials and methods) in a final volume $200 \mu \mathrm{l}$, giving a good band at $1500 \mathrm{bp}$ on agarose gel as shown in figure (xxx).The target band (1500 bp) excised, eluted and purified.

\section{Nucleotide sequence of alkaliphilic amylase gene}

The sequence of the obtained clone was determined on both strands by dideoxynucleotide triphosphate (ddNTP) chain termination method of (Sanger et al., 1977) using the automated sequence machine at Macrogen, South Korea. The obtained sequence was assembled, analyzed and compared with sequence in nucleotide database
(NCBI) using the BLAST algorithm. The entire open reading frame of amylase gene containing 1500 bases encodes a protein with (449) amino acids and a molecular mass ( 53 $\mathrm{KDa}$ ) and has been deposited in the GenBank database under accession number (KT693278) as shown in figure .

Characterization of alkaliphilic amylase

Optimum temperature: Alkaliphilic amylase from $B$. licheniformis strain $M K 7$ has a wide range of activity at different degrees of temperatures, but it has been found that the highest activity for amylase was determined at $37 \mathrm{C}^{\circ}$ which represents the optimum temperature as shown in figure (6).

\section{Optimum pH}

Results recorded in figure (7) show that the activity of the alkaliphilic amylase increased as the $\mathrm{pH}$ values increased up to $\mathrm{pH} 9$ which represents the optimum $\mathrm{pH}$.

\section{Effect of some metal ions}

Data in figure (8) show that amylase activity response to activators and inhibitors. It appeared that $\mathrm{Mg}^{2+,} \mathrm{K}^{+}$and EDTA increase the enzyme activity, whereas $\mathrm{Ag}^{+}, \mathrm{Zn}^{2+}$ and Urea cause the most inhibition of enzyme activity.

The genus Bacillus produces a large variety of extracellular enzymes, of which amylases are of particularly considerable industrial importance (Swain et al., 2006). Present study deals with the production condition optimization and partial purification of crude extracellular amylase produced by Bacillus licheniformis was able to hydrolyze starch showing zone of hydrolysis around the colonies on agar medium supplemented with soluble starch four amylotic bacterial colonies among 14 colonies which were positive for amylase production isolated from farm soils of Sadat City, Menofiya government, Egypt. The major factors involved in the biosynthesis of amylases include growth phase, type and concentration of chemicals in the growth media, as well as inducibility and repressibility of the enzyme (Ikram et al., 2002).

we designed primers for amylase genes from the genome of the closest strains having complete genome sequence. Amylase gene was amplified by PCR by using A strain of our lab stocks an alkaliphilic Bacillus licheniformis strain MK7 which have accession number (KP322016) was used. Three degenerative primers for Bacillus licheniformis was used, two primers for the whole amylase gene and two primers for about half the gene, one bands were obtained at the expected size in PCR amplified 


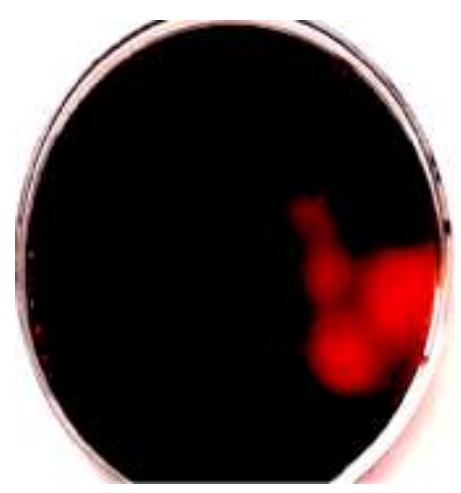

(Fig.1) showing amylotic activity of four bacterial

product of amylase gene from Bacillus licheniformis strain MK7 appeared at about $1500 \mathrm{bp}$ on the agrose gel. The target band (1500 bp) excised, eluted, purified and a part was sequenced at Macrogen, South Korea and compared with sequence in nucleotide database (NCBI) using the BLAST algorithm, Phylogenetic tree based on a comparison of the amino acid sequences of amylase licheniformis (WP_025807921.1), Bacillus licheniformis (ABF61440.1) and Bacillus licheniformis (AHJ11193.1).

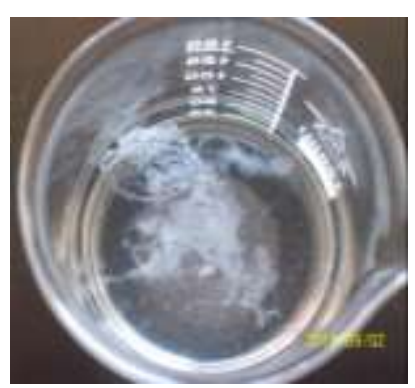

Figure. (2). Genomic DNA extraction $\begin{array}{lll}1 & M & 2\end{array}$

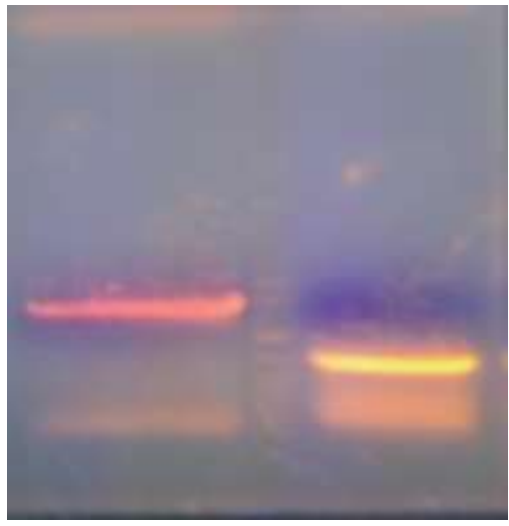

$1.5 \mathrm{kbp}$ $800 \mathrm{bp}$
1 $\begin{array}{lllllllllllllllllllllllllllll}P & \text { H } & \text { S } & \text { A } & \text { A } & \text { A } & \text { A } & \text { A } & \text { N } & \text { L } & \text { N } & \text { G } & \text { T } & \text { L } & \text { M } & \text { Q } & \text { Y } & \text { F } & \text { E } & \text { W } & \text { Y } & \text { M } & \text { P } & \text { N } & \text { D } & G\end{array}$

81 $\begin{array}{llllllllllllllllllllllllllll}Q & \text { H } & \text { W } & \text { K } & \text { R } & \text { L } & \text { Q } & \text { N } & \text { D } & \text { S } & \text { A } & \text { Y } & \text { L } & \text { A } & \text { E } & \text { H } & \text { G } & \text { I } & \text { T } & \text { A } & \text { V } & \text { W } & \text { I } & \text { P } & \text { P } & \text { A } & \text { Y }\end{array}$ AACATTGGAAGCGCTTGCAAAACGACTCGGCATATTTGGCTGAACACGGTATTACTGCCGTCTGGATTCCCCCGGCATAT

$\begin{array}{llllllllllllllllllllllllllllll}K & G & T & S & Q & A & D & V & G & Y & G & A & Y & D & \text { L } & Y & \text { D } & \text { L } & G & \text { E } & \text { F } & \text { H } & \text { Q } & \text { K } & G & \text { T } & \text { V }\end{array}$ 161 AAGGGAACGAGCCAAGCGGATGTGGGCTACGGTGCTTACGACCTTTATGATTTAGGGGAGTTTCATCAAAAAGGGACGGT

$\begin{array}{lllllllllllllllllllllllllllll}R & T & K & Y & G & T & K & G & E & \text { L } & \text { Q } & \text { S } & \text { A } & \text { I } & \text { K } & \text { S } & \text { L } & \text { H } & \text { S } & \text { R } & \text { D } & \text { I } & \text { N } & \text { V } & \text { Y } & G\end{array}$

241 TCGGACAAAGTACGGCACAAAAGGAGAGCTGCAATCTGCGATCAAAAGTCTTCATTCCCGCGACATTAACGTTTACGGGG $\begin{array}{lllllllllllllllllllllllllllll}\text { D } & \text { V } & \text { V } & \text { I } & \text { N } & \text { H } & \text { K } & \text { G } & G & \text { A } & \text { D } & \text { A } & \text { T } & \text { E } & \text { D } & \text { V } & \text { T } & \text { A } & \text { V } & \text { E } & \text { V } & \text { D } & \text { P } & \text { A } & \text { D } & \text { R } & \text { N }\end{array}$ 321 ATGTGGTCATCAACCACAAAGGCGGCGCTGATGCGACCGAAGATGTAACCGCGGTTGAAGTCGATCCCGCTGACCGCAAC $\begin{array}{lllllllllllllllllllllllllll}R & V & I & S & G & E & H & R & I & K & A & \text { W } & \text { T } & \text { H } & \text { F } & \text { H } & \text { F } & \text { P } & G & \text { R } & G & \text { S } & \text { T } & \text { Y } & \text { S } & \text { D } & F\end{array}$ 401 CGCGTAATTTCAGGAGAACACCGAATTAAAGCCTGGACACATTTTCATTTTCCGGGGCGCGGCAGCACATACAGCGATTT

$$
\begin{array}{lllllllllllllllllllllllllll}
\text { K } & \text { W } & \text { H } & \text { W } & \text { Y } & \text { H } & \text { F } & \text { D } & G & \text { T } & \text { D } & \text { W } & \text { D } & \text { E } & \text { S } & \text { R } & \text { K } & \text { L } & \text { N } & \text { R } & \text { I } & \text { Y } & \text { K } & \text { F } & \mathbf{Q} & G
\end{array}
$$
TAAATGGCATTGGTACCATTTTGACGGAACCGATTGGGACGAGTCCCGAAAGCTGAACCGCATCTATAAGTTTCAAGGAA

$\begin{array}{lllllllllllllllllllllllllllll}\text { K } & \text { A } & \text { W } & \text { D } & \text { W } & \text { E } & \text { V } & \text { S } & \text { N } & \text { E } & \text { N } & \text { G } & \text { N } & \text { Y } & \text { D } & \text { Y } & \text { L } & \text { M } & \text { Y } & \text { A } & \text { D } & \text { I } & \text { D } & \text { Y } & \text { D } & \text { H } & \text { P }\end{array}$ AGGCTTGGGATTGGGAAGTTTCCAATGAAAACGGCAACTATGATTATTTGATGTATGCCGACATCGATTATGACCATCCT TCCGAACCCTAACCCTTCAAAGGTTACTTTTGCCGTTGATACTAATAAACTACATACGGCTGTAGCTAATACTGGTAGGA
80

160

240 
$\begin{array}{lllllllllllllllllllllllllllll}\text { A } & E & \text { Y } & \text { W } & \text { Q } & \text { N } & \text { D } & \text { L } & G & \text { A } & \text { L } & \text { E } & \text { N } & \text { Y } & \text { L } & \text { N } & \text { K } & \text { T } & \text { N } & \text { F } & \text { N } & \text { H } & \text { S } & \text { V } & \text { F } & \text { D } & \text { V }\end{array}$ CTGAATATTGGCAGAATGACTTGGGCGCGCTGGAAAACTATTTGAACAAAACAAATTTTAATCATTCAGTGTTTGACGTG

$\begin{array}{llllllllllllllllllllllllllllll}P & \text { L } & \text { H } & \text { Y } & \text { Q } & \text { F } & \text { H } & \text { A } & \text { A } & \text { S } & \text { T } & \text { Q } & \text { G } & \text { G } & \text { G } & \text { Y } & \text { D } & \text { M } & \text { R } & \text { K } & \text { L } & \text { L } & \text { N } & \text { S } & \text { T } & \text { V } & \text { V }\end{array}$ CCGCTTCATTATCAGTTCCATGCTGCATCGACACAGGGAGGCGGCTATGATATGAGGAAATTGCTGAACAGTACGGTCGT

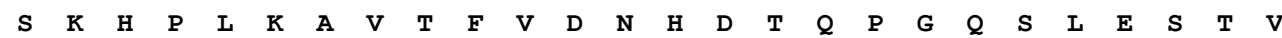

961 TTCCAAGCATCCGTTGAAAGCGGTTACATTTGTCGATAACCATGATACACAGCCGGGGCAATCGCTTGAGTCGACTGTCC

$\begin{array}{llllllllllllllllllllllllllll}Q & T & \text { W } & \text { F } & \text { K } & \text { P } & \text { L } & \text { A } & \text { Y } & \text { A } & \text { F } & \text { I } & \text { L } & \text { T } & \text { R } & \text { K } & \text { S } & \text { G } & \text { Y } & \text { P } & \text { H } & \text { V } & \text { F } & \text { S } & G & \text { D } & M\end{array}$

1041 AAACATGGTTTAAGCCGCTTGCTTACGCTTTTATTCTCACAAGGAAATCTGGATACCCTCACGTTTTCTCCGGGGATATG

$\begin{array}{llllllllllllllllllllllllllllll}\mathbf{Y} & G & \text { T } & \text { K } & \text { R } & \text { N } & \text { S } & \text { P } & \text { P } & \text { E } & \text { I } & \text { L } & \text { A } & \text { L } & \text { K } & \text { H } & \text { K } & \text { I } & \text { E } & \text { P } & \text { I } & \text { L } & \text { K } & \text { A } & \text { R } & \text { K } & Q\end{array}$

1121 TACGGGACGAAAAGAAACTCCCCCCCCGAAATTCTTGCCTTGAAACACAAAATTGAACCCATCTTAAAAGCGAGAAAACA

1121 ATGCCCTGCTTTTCTTTGAGGGGGGGGCTTTAAGAACGGAACTTTGTGTTTTAACTTGGGTAGAATTTTCGCTCTTTTGT

ATGCCCTGCTTICTTTGAGGGGGGGCTITAAGAACGGAACTTTGTGTTTIAACTTGGGTAGAATTTCGCTCITITGI

1201 GTATGCGTACGGAGCACAGCATGATTATTTCGACCACCATGACATTGTCGGCTGGACAAGGGAAGGCGACAGCTCGGTTG

$$
\begin{array}{lllllllllllllllllll}
\text { E } & \text { T } & \text { W } & \text { H } & \text { D } & \text { I } & \text { T } & G & \text { N } & \text { R } & \text { S } & \text { E } & \text { P } & \text { V } & \text { V } & \text { I }
\end{array}
$$

GAGACATGGCATGACATTACCGGAAACCGTTCGGAGCCGGTTGTCATC 1408

Fig 4. The sequence of acillus .licheniformis MK7 amyA gene and its deduced amino acid residues (in upper area), with restriction map for some restriction enzymes.

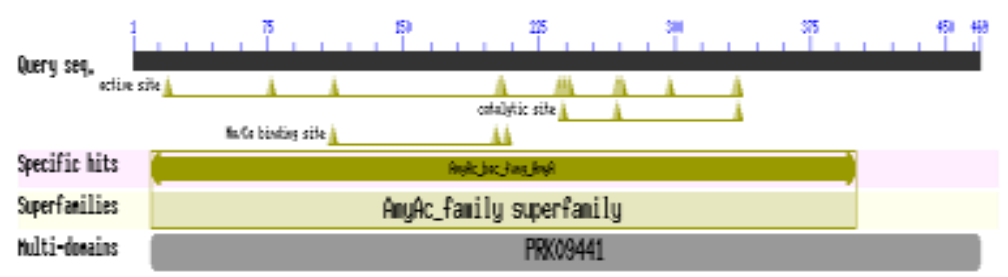

Fig 5. Putative conserved domains have been detected, click on the image below for detailed results.

AKI 30040.1

AJO16877.1 mk 0000000 ABF 61440.1 WP-025807921 AHJ11193.1

AKI 30040.1 AJO1 6877.1 mk 0000000 $\mathrm{AB} \overline{\mathrm{F}} 61440.1$ WP-025807921 AHJ11193.

AKI30040.1 AJO16877.1। mk_0000000
-MKQHKRLYARLLPLLFAL MRRKRH I GAFLLQENI GKT I FAKNSKYLYNSMCFTLKGERKIMKQHKRLYARLLPLLFAL

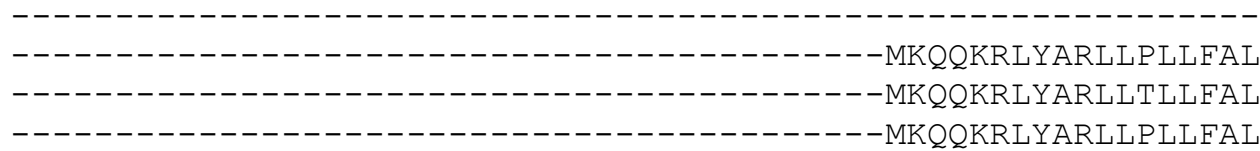

IFLLPHSAAAAASLNGTLMQYFEWYMPNDGQHWKRLQNDSAYLAEHGITAVWIPPAYKGT IFLLPHSAAAAASLNGTLMQYFEWYMPNDGQHWKRLQNDSAYLAEHGITAVWI PPAYKGT ----PHSAAAAANLNGTLMQY FEWYMPNDGQHWKRLQNDSAYLAEHGITAVWIPPAYKGT IFLLPHSAAAAANLKGTLMQYFEWYMPNDGQHWKRLQNDSAYLAEHGITAVW I PPAYKGT IFLLPHSAAAAANLNGTLMQYFEWYMPNDGQHWKRLQNDSAYLAEHGITAVW I PPAYKGT IFLLPHSAAAAANLKGTLMQYFEWYMPNDGQHWKRLQNDSAYLAEHGITAVWI PPAYKGT

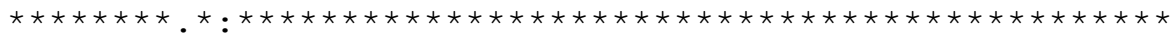

SQDDVGYGAYDLYDLGEFHQKGTVRTKYGTKGELQSA INSLHSRDINVYGDVVINHKGGA SQDDVGYGAYDLYDLGEFHQKGTVRTKYGTKGELQSA INSLHSRDINVYGDVVINHKGGA SQADVGYGAYDLYDLGEFHQKGTVRTKYGTKGELQSA IKSLHSRDINVYGDVV INHKGGA 
ABF 61440.1 WP- 025807921 AHJ11193.

AKI 30040.1 AJO16877.1 mk 0000000 $\mathrm{AB} \overline{\mathrm{F}} 61440.1$ WP-025807921 AHJ11193.

AKI 30040.1 AJO16877.1 $\mathrm{mk} 0000000$ $\mathrm{AB} \overline{\mathrm{F}} 61440.1$ WP-025807921 AHJ11193.

AKI 30040.1 AJO16877.1 mk 0000000 $\mathrm{AB} \overline{\mathrm{F}} 61440.1$ । WP-025807921 AHJ11193.|
SQADVGYGAYDLYDLGEFHQKGTVRTKYGTKGELQSA IKSLHSRDINVYGDVVITNKGGA SQADVGYGAYDLYDLGEFHQKGTVRTKYGTKGELQSA IKSLHSRDINVYGDVVINHKGGA SQADVGYGAYDLYDLGEFHQKGTVRTKYGTKGELQSAIKSLHSRDINVYGDVVINHKGGA

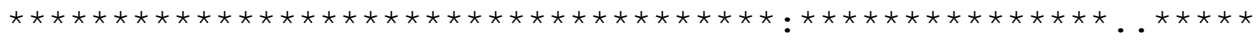

FILTREAGYPQIFYGDMYGTKGASQREIPALKHKIEPILKARKQYAYGAQHDYFDHHDIV FILTREAGYPQIFYGDMYGTKGASQREIPALKHKIEP ILKARKQYAYGAQHDYFDHHNIV FILTRKSGYPHVFSGDMYGTKRNSPPEILALKHKIEP ILKARKQYAYGAQHDYFDHHDIV FILTRESGYPSGFYGDMYGTKGASQREIPALKHKIEPILKARKQYAYGAQHDYFDHHDIV FILTRESGYPQVFYGDMYGTKGDSQREIPALKHKIEPILKARKQYAYGAQHDYFDHHDIV FILTRESGYPQVFYGDMYGTKGDSQREIPALKHKIEPILKARKQYAYGAQHDYFDHHD--

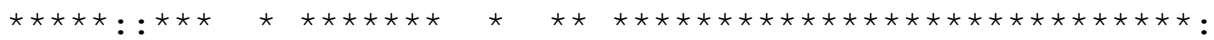

GWTREGDSSVANSGLAALITDGPGGTKRMYVGRQNAGETWHDITGNRSDSVVINAEGWGE GWTREGDSSVANSGLAALITDGPGGTKRMYVGRQNAGETWHDITGNRSDSVVINAEGWGE GWTREGDSSVANSGLAALITDGPGGA-RMYVGRQNAGETWHDITGNRSEPVVI------GWTREGDSSVANSGLAALITDGPGGAKRMYVGRQNAGETWHDITGNRSEPVVINSEGWGE GWTREGDSSVANSGLAALITDGPGGAKRMYVGRQNAGETWHDITGNRSEPVVINSEGWGE

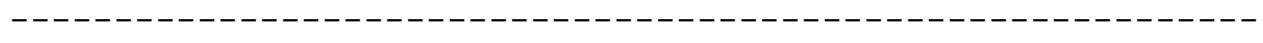

FHVNGGSVS IYVQR
FHVNGGSVS IYVQR
--------------
FHVNGGSVS IYVQR
FHVNGGSVS IYVQR
--------------

Fig 6. Multiple deduced amino acid sequence alignment of amylase gene (KT693278) from B. licheniformis strain-MK7 with other reported, Bacillus licheniformis(AKI30040), Bacillus licheniformis(AJO16877), Bacillus licheniformis (WP025807921),Bacillus licheniformis (ABF61440) and Bacillus licheniformis (AHJ11193)

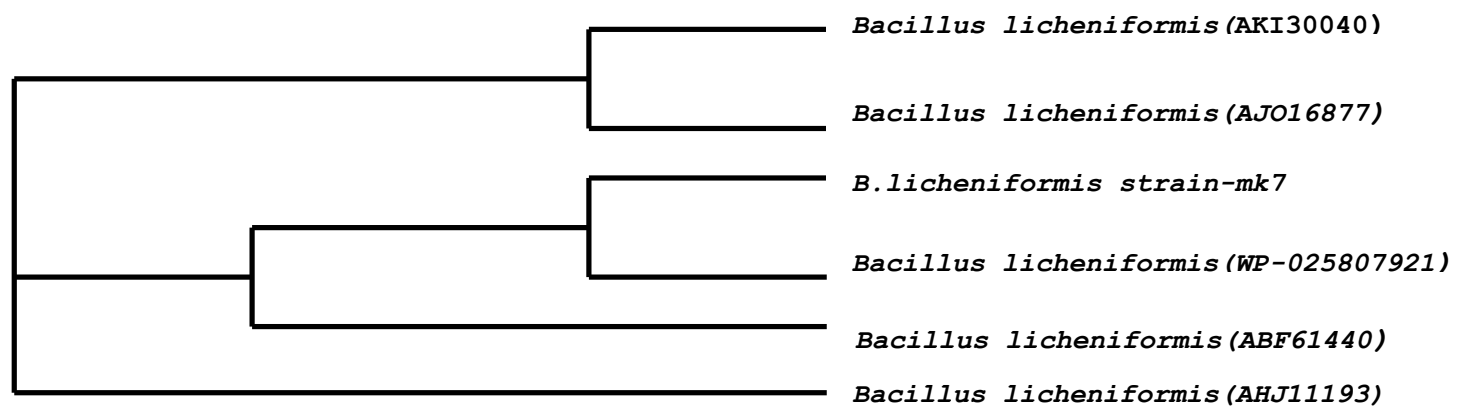

Fig 7. Phylogenetic tree based on a comparison of the amino acid sequences of amylase producing bacterial isolate $B$. licheniformis strain-MK7 and some of their closest phylogenetic relatives, Phylogenetic tree based on a comparison of the amino acid sequences of amylase producing bacterial isolate B. licheniformis strain-MK7 and some of their closest phylogenetic relatives Bacillus licheniformis(AKI30040), Bacillus licheniformis(AJ016877), Bacillus licheniformis (WP025807921),Bacillus licheniformis (ABF61440) and Bacillus licheniformis (AHJ11193) 


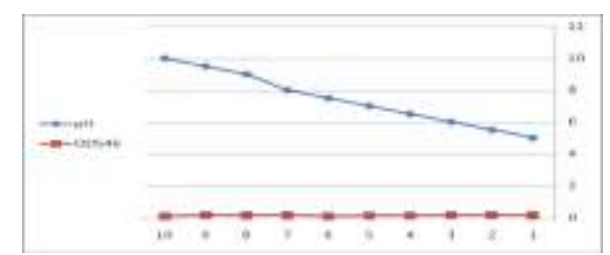

(Fig.8) shows effect of differenet $\mathrm{PH}$ on Amylase activity.

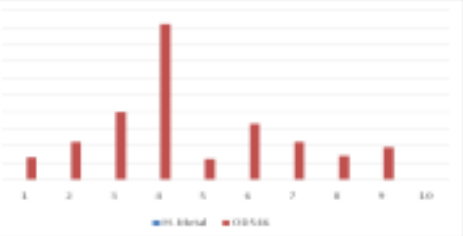

(Fig.10) Shows effect of different metal ions on amylase activity.

The second part of amylase gene purified was used for cloning the gene in TA cloning, we tried to clone the AmyA several time into two different TA clonning vectors from promega (PGEM) and ferments but there's no gene transfers this may be the longer size of PCR fragment. The amylase gene of Bacillus licheniformis strain-mk7 was amplified by PCR technique using consensus primer sequences found in closely related bacteria, the resultant amplicon, did show the presence of a signal sequence.When the sequence was analyzed by the GENETYX,it was predicated that the encoded amylase enzyme could be secreted through the extracellular medium of lichenniformis mk7. The amylase gene of Bacillus licheniformis strain - $\mathrm{mk} 7$ and other amylases from two alkaliphilic amylolytic isolates (ksm1and ksm2) were identified according to the methods in Bergey's Manual of Systematic Bacteriology and showed rod-shaped morphology; Gram positive reaction, catalase positive showed that they were long Bacillus sp. Sequencing of amylase gene of Bacillus licheniformis strain MK7 has been completed by using three degenerative oligonucleotide primers whose sequences were designed on the basis of amylase sequences of Bacillus licheniformis strain DSM13 to get the whole gene sequence open reading frame (ORF). The nucleotide sequence of the alkaliphilic amylase gene (AmyA) was deposited to the GenBank under accession number (KT693278). The deduced amino acids sequence derived from the open reading frame of AmyA was analyzed. The mature protein

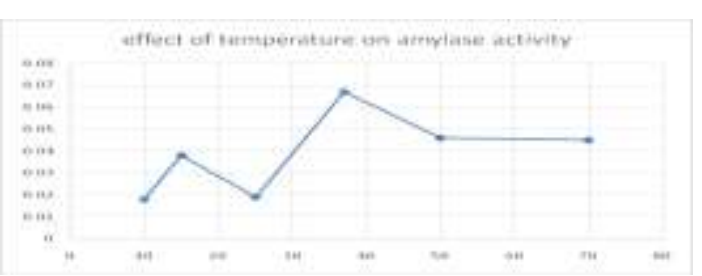

(Fig.9) shows effect of temperature difference on Amylase activitv.

Table 1. Amino acid composition of amylase A of Bacillus licheniformis strain-mk7

\begin{tabular}{|l|l|l|}
\hline Amino Acid & Number & Mol\% \\
\hline Ala A & 39 & 8.32 \\
\hline Cys C & 0 & 0.00 \\
\hline Asp D & 36 & 7.68 \\
\hline Glu E & 22 & 4.69 \\
\hline Phe F & 19 & 4.05 \\
\hline Gly G & 40 & 8.53 \\
\hline Ile I & 19 & 4.05 \\
\hline Lys K & 29 & 6.18 \\
\hline Leu L & 29 & 6.18 \\
\hline Met M & 7 & 1.49 \\
\hline Asn N & 24 & 5.12 \\
\hline Pro P & 17 & 3.62 \\
\hline Gln Q & 17 & 3.62 \\
\hline Arg R & 21 & 4.48 \\
\hline Ser S & 25 & 5.33 \\
\hline Thr T & 27 & 5.76 \\
\hline Val V & 29 & 6.18 \\
\hline Trp W & 16 & 3.41 \\
\hline Tyr Y & 28 & 5.97 \\
\hline
\end{tabular}

was 449 amino acids in length and has a calculated molecular mass of $55 \mathrm{KDa}$. Similarity searches performed with Basic Local Alignment Search Tool (BLAST http://www.ebi.ac.uk/Tools/sss/wublast/) using the deduced amino acid sequence of AmyA as the query

revealed its belonging to family $12 \mathrm{~A}$ enzymes (http://www.cazy.org/12A.html; extracted from the carbohydrate-active enzymes database (http://www.cazy.org/, (Cantarel et al., 2009). Figure (19) showing effect of temperatures on amylase activity, it was found that enzyme exhibited maximum activity at $37^{\circ} \mathrm{C}$. When the temperature increased above $37^{\circ} \mathrm{C}$, enzyme activity was affected negatively and gradually reduced. In the same way, when the temperature was below $37^{\circ} \mathrm{C}$, a gradual decline in the amylase activity was observed. Various Bacillus species are produced amylase enzyme at a wide range of temperatures. Bacillus amyloliquefaciens, 
Bacillus subtilis, Bacillus licheniformis and Bacillus stearothermophilus are among the most commonly used Bacillus species reported to produce $\alpha$-amylase at temperatures of $37-60^{\circ} \mathrm{C}$ (Mendu et al.,2005b; Mishera et al., 2005a; Syu and Chen, 1997;Mielenz, 1983). The optimum temperature of the $\alpha$-amylase is $37^{\circ} \mathrm{C}$, with a loss of activity over $70^{\circ} \mathrm{C}$. This property might limit industrial applications of the enzyme at high temperatures, but favors its application in processes that require complete inactivation of the enzyme, such as the baking industry (Coronado et al., 2000). The broad range of temperatures and the enzyme's high activity at both moderate and lower temperature values make this enzyme highly attractive for both basic research studies and industrial processes. A modern trend among consumers is to use colder temperatures for laundry or dishwashing. At lower temperatures, the removal of starch from cloth and porcelain becomes more problematic. Detergents with $\alpha$-amylases working optimally at low or moderate temperatures can overcome this problem (Van der Maarel et al., 2002).Figure (20) reflects the effect of $\mathrm{pH}$ on amylase activity. In fact $\mathrm{pH}$ affects the enzyme activity it showed that $\mathrm{pH} 9$ enhance amylase activity to reach its maximum. Therefore we could say that $\mathrm{pH} 9$ is the optimum $\mathrm{pH}$ for amylase activity. $B$. subtilis, $B$. licheniformis, and B.amyloliquefaciens, like Bacillus cereus GUF8, require an initial pH of 7.0 for maximal amylase production (Haq et al., 2005a; Tanyildizi, et al., 2005b; Syu and Chen, 1997). The archaeal pullulanases reported to date show maximal activity in the $\mathrm{pH}$ range 5.0 to 6.5 , with the exception of pullulanase type II from Pyrodictium abyssi, which exhibits its highest activity at pH 9.0 (Bertoldo and Antranikian. 2002). In accordance with the acidic catalysis mechanism proposed for amylolytic enzymes (Kim et al., 1999), the recombinant TK-PUL has an optimum pH of 3.5 in sodium acetate and 4.2 in sodium citrate buffer and is active over a broad $\mathrm{pH}$ range (3.0 to 8.5). $\alpha$-amylase purified from $B$. cereus GUF8 appeared as a single polypeptide with a molecular weight of about $56 \mathrm{kDa}$ similar to $B$. licheniformis and $B$. amyloliquefaciens $\alpha$-amylases. The enzyme activity was measured in presence of various ions at a final concentration of $1 \mathrm{mM}$ in the reaction solution. Of all the metal ions tested, $\mathrm{Ag}^{+}$, Urea and $\mathrm{Zn}^{2+}$ showed remarkable inhibitory effects because they reduced the enzyme activity by $64 \%, 42 \%$ and $12 \%$ respectively
(Fig.21) but other metals as Co, EDTA and $\mathrm{K}^{+}$showed stimulating effects of $34 \%, 19.2 \%$ and $14 \%$, respectively. The results are in close agreement with the study of recombinant expression of amylase from Bacillus subtilis in E. coli by (Zafar ., et al. 2014), that showed increase in the enzyme activity by Co but amylase activity was inhibited by $\mathrm{Zn}^{2+}, \mathrm{Hg}^{2+}$ and $\mathrm{Ni}^{2+}$ ions. Although it is difficult to compare the kinetic values of $\alpha$-amylases reported by other groups in view of the different starch substrates and their assay conditions, the $K m$ value of $B$. cereus GUF8 $\alpha$ amylase, $1.4 \mathrm{mg} / \mathrm{ml}$, is within the range of majority of other $\alpha$-amylases $(0.35$ to $4.7 \mathrm{mg} / \mathrm{ml}$ ) (Aguilar et al., 2000). The hydrolysis products of soluble starch were determined by TLC analysis. The major end products of the enzyme were G2-G5 together with smaller amounts of higher maltooligosaccharides, however, it was not able to produce glucose, These indicate that the amylolytic enzyme from $B$. cereus GUF8 hydrolyzes starch by randomly cleaving internal $\alpha-1,4-$ glycosidic linkages and therefore the reaction proceeds in an endo-type fashion (Mezghani and Bejar. 1999; Prieto et al., 1995).Industrial starch hydrolysis is a two-step process, amylase and pullulanase is normally added during the second step (saccharification) to hydrolyze $\alpha-1,6$ linkages at branch points that were not attacked by $\alpha$-amylase in the first step (liquefaction) (Lévêque et al. 2000). Complete solubilization of starch in water can only be achieved at a temperature above $100^{\circ} \mathrm{C}$, and the natural $\mathrm{pH}$ of this solution is 4.5. Therefore, the use of highly thermostable amylolytic enzymes capable of working efficiently in this acidic environment would directly benefit the starch industry (Van der Maarel, 2002.). We describe here alkaliphilic amylase -hydrolyzing enzyme from Bacillus. licheniformus The amino acid sequence of TK-PUL displayed homology with type II pullulanases, and it was annotated accordingly during the analysis of the gene sequence of Bacillus. licheniformus (Fukui et al ., 2005). A major problem for the starch industry is that $\mathrm{Ca}^{+2}$ has to be added to starch slurry to enhance the thermostability and activity of amylases. This added $\mathrm{Ca}^{+2}$ inhibits the activity of glucose isomerase, which is used in later steps for isomerization of glucose to fructose during highfructose syrup production (Wang et al., 2007). Calcium oxalate is also produced as a waste product and deposits in the pipes and heat exchangers. This deposition chokes the pipes and in turn increases the production cost. With 
the development of $\mathrm{Ca}^{+2}$ independent thermostable enzymes, this problem can be solved. AmyA does not need $\mathrm{Ca}^{+2}$ for activity and thermostability. Its $\mathrm{Ca}^{+2}$ independence, along with its high thermostability and $\mathrm{pH}$ optima of 9.0 in buffer, make AmyA an attractive candidate for application in the starch industry.As both the $\alpha-1,4-$ and $\alpha-1,6$-hydrolyzing activities of recombinant Amylase $A$ was inhibited at the same rate in the presence of $0.1 \%$ cyclodextrin, $0.01 \% \quad p$ chloromercuribenzoic acid, and $0.01 \% \quad \mathrm{~N}$ bromosuccinimide, it can be speculated that recombinant AmyA possesses a single active site for the hydrolysis of both $\alpha-1,4$ and $\alpha-1,6$ glucosidic linkages. However, detailed studies are required to reach such a conclusion. Amylopullulanases from D. mucosus (Duffner et al., 2000.), Thermoanaerobium strain Tok6-B1 (Plant et al., 1987), and C. thermohydrosulfuricum (Mathupala et al., 1990) have been shown to possess one active site that hydrolyzing both 1,4 linkage and 1,6 linkage, whereas amylopullulanases $\mathrm{N}$ from Bacillus circulans F-2 (Kim and Kim . 1995), Bacillus sp. strain KSM- 1378 (Hatada et al., 1996) and $P$. woesei (Rudiger et al., 1995) have been reported to possess two different active sites responsible for dual catalytic activities. The amylase activity was slightly inhibited in the presence, possibly due to substrate competition. The slight inhibition may be attributed to a possible catalytic site being involved in both types of reactions. Among previously reported amylases, only two enzymes, from $T$. aggregans (Niehaus et al., 2000) and D. mucosus (MacGregor et al., 2001), were able to hydrolyze cyclodextrins, while others were competitively inhibited. AmyA was able to hydrolyze Amylose into glucose. These annotations were based on the amino acid sequence comparisons. Sequence-based classification of putative enzymes may not always be correct, as observed in the case of the well-characterized branching enzyme TK1436 from T. kodakarensis. TK1436 was annotated as a "probable $\alpha$-amylase" in the genome sequence, but the biochemical characterization was in contrast with the sequence-based annotation and showed that it was a branching enzyme (Murakami et al., 2006).

\section{REFERENCES}

ahajan R., Manhas M., Priya B., Kaur K. and Kaur G. Research Journal of Pharmaceutical, Biological and.....amo G., Gashe B.A. and Gessesse A. 1999. A highly thermo-stable amylase from a newly isolated thermophilic Bacillus sp. WN11. J. Appl. Microbial. 86:557-560.

ambare V. 2010a. Solid state fermentation of aspergillus oryzae for glucoamylase production on agro residues. Int. J .life sci. 4:16 25.

arikaya E., Higassa T., Adachi M. and Mikami B. 2000. Proc. Biochem. 35: 711-715.

candurra R., Consalvi, chiaraluce R., Politi L. and Engel P.C. (2000). Front Biosic. 5: 787-795.

choudhary A., Mehta D.S., Biradar A.M. 2009. phys.Rev.E.stat.Nonlinear soft matter phys.80:12701.

eddy N.S., Nimmagadda S.A. and Rao K. R.S. 2003a. An over view of the microbial alpha amylase family. A fr. J. Biotechnol. 2:645 -648.

han J.A. and Priya R. 2011. Advances in Applied Science Research 2 (3): 509-519.

harani, A. P. V. 2004. Effect of C: $\mathrm{N}$ ratio on alpha amylase production by Bacillus licheniformis SPT 27. Afr .J .Biotechnol. 3:519 -522.

hasemi M., Shafaroodi H.and Nazarbeikis. 2010. voltage dependent calcium channel and NMDA receptor antagonists augment anticonvulsant effects of lithium chloride on pentylenetet razole. Induced clonic seizures in mice .Epilepsy Behave 18(3):171178.

herry, H.M., Hossain, M. T. and Anwar, M.N. 2004. Extracellular glucoamylase from the isolate Aspergillus fumigatus. Pakit. J. Biol. Sci. 7 (11): 19881992.

iller G. L.1959. Use of dinitrosalicylic acid reagent for determination of reducing sugar. Analy.l Chem.31:426-428.

indhu M.K., Singh B.K.and Prased T. 1997. In. Phytopathol., 34: 269-271.

isher EH. And Stein EA. 1961. Biochem. Prepn. 8: 27-32.

K Lonsane and Ramesh M.V. Advances in Appl. Microbial., 1990, 35: 54-56.Chemical Science, 2011, 2(4), 239246. chemical treated mutant of Bacillus subtilis. J. Biol. Sci. 2(2): 73-75.

Komaki T. and Tonomura K.1993. Purification and Properties of Extracellular 6-Mannanases Produced by Enterococcus casseliflavus FL2121 Isolated from Decayed Konjac. J. Ferment. and Bioengin. 76:14-18. 
kram H., Rani S., Ashraf H. and Qadeer M. A. 2002. Biosynthesis of 4-amylase by International (P) Ltd., Publishers, New Delhi, 2003; pp 320.

Moreau Y., Santimone M., Svensson B., Marchis-Mouren G. and Desseaux V. 2003. On the mechanism of $\alpha$ amylase: Acarbose and cyclodextrin inhibition of barley.

nderkofler L.A. and Hickey R.J. Industrial fermentations, Vol. II, Chemical Publishing Co., New York, 1954; pp.

Nigama p., Carlos R. S., Vanete T. S., Dalel S. and M. A. 2000. Advances in microbial amylases.Biotechnolol.Appl. Biochem.

oung M. H., Dong G.L., Jung-Hoon Y., Yong-Ha P. and.Young J. K. 2001. Rapid and simple purification of a novel extracellular beta - amylase from Bacillus sp . Biotechnol. lett. 23:1435-1438.

Sambrook J., Russel D. W. 2001. Molecular Cloning: A Laboratory Manual, Volume 1, 2, 3.Cold Spring Harbour Laboratory Press, Cold Spring Harbour, New York.

Sanger F., Nicklen S., and Coulson A. R. 1977. DNA sequencing with chain-terminating inhibitors. Proc. Natl. Acad. Sci. U S A. 74(12): 5463-5467.

Takahashi A. and Makoto S. 1996. Isolation of a gene essential for biosynthesis of the lipopeptide antibiotics plipastatin B1 and surfactin in Bacillus subtilis YB8. Archives of Microbiology 165: 243-251.
Tankasale A., Ghatge M. and Desphande V. Microbial. 1998. Mol. Biol. Rev. 62: 597-634.

Tapan kumar D. and Chandra R.R. 2010.Optimization of thermo-stable alpha amylase production by Streptomyces erumpens MTCC 7317 in solid state fermentation using cassava fibrous residue. Brazilian Arch. Boil. Technol.53:301-309.

udharhsan S., Senthilkumar S. and Ranjith K. 2007. Afr. J. Biotechnol., 6(4): 430-435.

upta R., Gigras P., Mohapatra H., Goswami V. K. and Chauhan B. 2003. Microbial alpha amylase: a biotechnological perspective, Process Biochemistry 38: 1599-1616.

wain M.R., Kar S., Padmaja G. and Ray R.C. 2006. Partial characterization and optimization of production of extracellular $\alpha$-amylase from Bacillus subtilis isolated from culture able cow dung microflora. Pol. J. Microbial 55(4):289-296.

Yuguo Z., Zhao W. and Xiaolong C.2000.Alpha amylase production by Bacillus subtilis with dregs in an external-loop airlift bioreactor. Biochem. Eng .J. 5:115-121. 